\title{
A cluster randomised control trial of a multi-component weight management programme for adults with intellectual disabilities and obesity
}

\author{
Leanne Harris ${ }^{1}$, Catherine Hankey ${ }^{2}$, Nathalie Jones ${ }^{1}$, Carol Pert ${ }^{3}$, Heather Murray ${ }^{4}$, Janet Tobin ${ }^{5}$, \\ Susan Boyle ${ }^{6}$ and Craig Melville ${ }^{1 *}$ \\ ${ }^{1}$ College of Medical Veterinary and Life Sciences, Institute of Mental Health E Wellbeing, University of Glasgow, \\ Glasgow G12 OXH, UK \\ ${ }^{2}$ College of Medical Veterinary and Life Sciences, Institute of Health \& Wellbeing, University of Glasgow, Glasgow G31 2ER, UK \\ ${ }^{3}$ Learning Disability Psychology, National Health Service (NHS) Greater Glasgow and Clyde, Glasgow G52 $2 H H$, UK \\ ${ }^{4}$ Robertson Centre for Biostatistics, Institute of Health and Wellbeing, University of Glasgow, Glasgow G12 8QQ, UK \\ ${ }^{5}$ Glasgow City Community Health Partnership (CHP) North East Sector, Eastbank Conference Training Centre, Glasgow G32 9 AA, UK \\ ${ }^{6}$ Glasgow and Clyde Weight Management Service, Glasgow G3 8SJ, UK
}

(Submitted 6 February 2017 - Final revision received 29 April 2017 - Accepted 17 June 2017)

\section{Abstract}

There have been few published controlled studies of multi-component weight management programmes that include an energy deficit diet (EDD), for adults with intellectual disabilities and obesity. The objective of this study was to conduct a single-blind, cluster randomised controlled trial comparing a multi-component weight management programme to a health education programme. Participants were randomised to either TAKE 5, which included an EDD or Waist Winners Too (WWToo), based on health education principles. Outcomes measured at baseline, 6 months (after a weight loss phase) and 12 months (after a 6-month weight maintenance phase), by a researcher blinded to treatment allocation, included: weight; BMI; waist circumference; physical activity; sedentary behaviour and health-related quality of life. The recruitment strategy was effective with fifty participants successfully recruited. Both programmes were acceptable to adults with intellectual disabilities, evidenced by high retention rates (90\%). Exploratory efficacy analysis revealed that at 12 months there was a trend for more participants in TAKE $5(50.0 \%)$ to achieve a clinically important weight loss of 5-10\%, in comparison to WWToO (20.8 \%) (OR 3.76; $95 \%$ CI $0.92,15 \cdot 30 ; 0 \cdot 064)$. This study found that a multi-component weight management programme that included an EDD, is feasible and an acceptable approach to weight loss when tailored to meet the needs of adults with intellectual disabilities and obesity.

Key words: Obesity: Weight management: Intellectual disabilities: TAKE 5

International clinical guidelines recommend multi-component weight management programmes as the treatment of choice in challenging the burgeoning obesity epidemic ${ }^{(1-4)}$. Multi-component weight management programmes have been shown to support sustainable weight losses of $5-10 \%$ body weight and associated with clinical improvements in reducing health risk factors ${ }^{(2,3)}$. Effective components primarily include a personalised energy deficit diet (EDD) involving a $2514 \mathrm{~kJ}$ $(600 \mathrm{kcal})$ reduction in total energy intake per day, support to increase physical activity and behaviour change techniques ${ }^{(2,3,5)}$.

Adults with intellectual disabilities (defined as a significant impairment in intellectual functioning and adaptive behaviour including practical and social skills $)^{(6)}$ have been reported to have a higher prevalence of obesity and associated health inequalities in comparison to the general population ${ }^{(7-10)}$. Despite adverse effects of obesity on the health of adults with intellectual disabilities, little is known about whether evidenced based approaches to weight management are effective for this population group. The current evidence has identified a paucity of weight management programmes for adults with intellectual disabilities $^{(11-13)}$. In a recent review ${ }^{(13)}$, only eight studies were classified as multi-component and were primarily focused on a health education approach, with only one offering participants an $\mathrm{EDD}^{(14)}$. The effect sizes of these studies were small and nonsignificant, and studies were limited in their reporting of clinical effectiveness. Furthermore, studies were of poor methodological quality, and only one study including a multi-component programme implemented a randomised control trial design ${ }^{(15)}$.

To fill the gap in current knowledge, TAKE 5 was developed and piloted ${ }^{(16-18)}$. TAKE 5 is a multi-component weight management programme developed to reflect UK weight management guidelines ${ }^{(2,3)}$. TAKE 5 was modelled on the approach

Abbreviations: EDD; energy deficit diet; EQ-5D; European Quality of Life-5 dimensions; IPAQ-S; International Physical Activity Questionnaire-Short; QOL; quality of life; WWToo; Waist Winners Too.

* Corresponding author: Dr C. Melville, fax +44 141211 0356, email Craig.Melville@glasgow.ac.uk 
used by a weight management service in the UK, the Glasgow and Clyde Weight Management Service (GCWMS) ${ }^{(19,20)}$. The approach used by GCWMS, developed for the general population, was adapted for adults with intellectual disabilities and implemented with support from participants' carers where applicable. Results of the single-stranded study illustrated that TAKE 5 was acceptable to adults with intellectual disabilities and their carers and demonstrated significant reductions in health risk factors including weight, BMI, waist circumference, sedentary behaviour and increased physical activity levels ${ }^{(16-18)}$. Based on these findings and initial support for a multi-component programme utilising an EDD in adults with intellectual disabilities, the aim of this study was to carry out a cluster randomised controlled trial of TAKE 5 in comparison to a health education programme (providing information on diet and physical activity behaviours).

\section{Methods \\ Study design}

This study was designed as a pilot, single-centre, single-blind randomised trial. Treatment as usual (TAU) for adults with intellectual disabilities in the UK, varies from no weight management programmes to community-based health education programmes. Therefore, to provide a standardised TAU, the Waist Winners Too (WWToo) health education programme was used as the control arm of the study ${ }^{(21)}$. Participants were randomised to TAKE 5 or WWTOO for a 12-month period; a 6-month weight loss period followed by a 6-month weight maintenance period. The trial was registered before data collection (http://www.isrctn.com/ ISRCTN52903778) and the study protocol has previously been published ${ }^{(22)}$. The trial is reported according to CONSORT guidelines for reporting cluster randomised designs ${ }^{(23)}$ and followed guidelines by the Medical Research Council on developing and evaluating 'complex' interventions ${ }^{(24,25)}$.

\section{Ethical approval}

The study was conducted in accordance with the ethical guidelines of the Declaration of Helsinki and consistent with the principles of Good Clinical Practice. Ethical approval was received from the Scotland A Research Ethics Committee (reference no.: 13/SS/0229) on 16 December 2013. The process of obtaining consent was conducted in accordance with the Adults with Incapacity Act, $2000^{(26)}$. Written informed consent was obtained from participants who had capacity. In circumstances where a participant did not have capacity, written informed consent was provided by the nearest relative or welfare guardian.

\section{Study population}

A multi-point recruitment strategy developed by Foster et $a l^{(27)}$ was utilised to recruit participants from multiple organisations in Greater Glasgow \& Clyde, Scotland, including specialist intellectual disabilities services, provider organisations and local day centres. Participants were eligible to take part if they were diagnosed with intellectual disabilities (all level of intellectual disabilities were included, mild to profound), adults ( $\geq 18$ years), obese (BMI $\geq 30 \mathrm{~kg} / \mathrm{m}^{2}$ ), ambulatory, not currently on a prescribed or restricted diet (e.g. for phenylketonuria or diabetes) and had not intentionally lost weight of $>3 \mathrm{~kg}$ in the previous 3 months. Participants were excluded if they had the following genetic syndromes; Prader-Willi syndrome, Cohen syndrome or Bardet-Biedl syndrome, taking medication for the purpose of losing weight (either prescribed or over the counter) and individuals who were pregnant or became pregnant during the study.

\section{Randomisation}

Cluster randomisation was conducted to minimise potential risk of contamination between programmes, clustering of outcomes and to minimise imbalance between study groups. If participants lived together (in shared tenancies) or were supported by the same group of paid carers they were randomised in a cluster. Participants were stratified by presence of Down's syndrome, level of intellectual disabilities and number of participants within a cluster. Randomisation was implemented by the researcher (L. H.) using an interactive voice response system. This was hosted by the Robertson Centre for Biostatistics, University of Glasgow. The researcher was blinded to participant group allocation. Participants were enrolled in the study through notification of the principal investigator via email of the group allocation.

\section{Sample size}

In the single-stranded pilot study, the sample size and recruitment were based on the referral of adults with intellectual disabilities to specialised intellectual disabilities dietitians ${ }^{(16)}$. The sample size for this study was calculated separately based on the feasibility of conducting a full-scale trial and to provide insight into recruitment and retention rates, which would have a $95 \% \mathrm{CI}$ of no more than $\pm 10 \%$; to determine the probable variance of outcomes to power a larger trial. If fifty participants provide 12-month outcome data, a $90 \%$ CI for each variance estimate would have a width of approximately $70 \%$ of the estimate (i.e. -29 to $+41 \%$ ); and to allow an attrition rate of $20 \%$. Before this study, there were no cluster randomised controlled trials of weight management programmes to inform the degree of clustering. Therefore, the sample size was increased by $10 \%$ based on the following assumption of a conservative intra-class correlation coefficient (ICC) of $0 \cdot 1$, and an average of two participants per cluster. Thus, the final samples size was projected to be sixty-six participants (thirty-three participants in each programme).

\section{Weight management programmes}

The two weight management programmes under investigation were both made up of a diet, physical activity and behaviour change components. The distinct difference between the two programmes was based on the inclusion of the quantitative EDD in the TAKE 5 programme. However, the diet component in the WWToo programme was based on general advice, with which compliance cannot be quantified. The physical activity 
component of the TAKE 5 programme is more comprehensive as it included the use of pedometers. Finally, the behaviour change techniques utilised in the TAKE 5 programme were more numerous and used more flexibly to meet the individual needs of participants.

A dietitian and a health professional, trained for the purpose of this study, delivered both programmes. Both had experience in delivering lifestyle programmes to adults with intellectual disabilities. To ensure fidelity and minimise contamination between the programmes, the dietitian and health professional followed a pre-set protocol and manual for each session. Weight management programmes were delivered on a one-to-one basis in the participant's home or convenient location. The programme sessions were delivered over a 12-month period, nine to twelve sessions in the weight loss phase and six sessions in the weight maintenance phase. The variability in the number of weight loss sessions was to allow appointments to be organised flexibly to maximise the consistent involvement of family and paid carers. Nine weight loss sessions were attended by carers and participants and no additional appointments were requested. Each session was scheduled to last approximately $40-60 \mathrm{~min}$. This was to allow appointments to be organised flexibly to account for individual needs and abilities of participants. Full details of the programmes have been published previously ${ }^{(15,17,21)}$.

\section{Carer involvement}

Participants were invited to be supported throughout the programmes by family and/or paid carers. Carers were invited to attend sessions to help with communication or where necessary the implementation of behaviour change techniques, for example, goal setting and self-monitoring of the participants' physical activity or dietary intake. The level of carer involvement was dependent on the individual needs and abilities of participants and ranged from minimal support for example assistance with completing food diaries or encouraging participation in physical activity, to 24-h support in preparing and cooking all meals and actively assisting the participant to go for a walk.

\section{TAKE 5 components}

\section{Diet}

To achieve a healthy sustainable weight loss of $0.5-1.0 \mathrm{~kg} /$ week participants followed a personalised EDD with a deficit of $2510 \mathrm{~kJ} / \mathrm{d}$ $(600 \mathrm{kcal} / \mathrm{d})^{(2,3)}$. Daily energy intake was calculated based on total energy requirements minus $2510 \mathrm{~kJ} / \mathrm{d}(600 \mathrm{kcal} / \mathrm{d})$. Participants total energy requirements were calculated based on their BMR (using the Mifflin St. Jeor equation) ${ }^{(28)}$ multiplied by a physical activity level of $1 \cdot 3^{(29)}$. Quantitative dietary intake was from a specified number of portions based on the EatWell Plate ${ }^{(30)}$ and based on recommendations of a healthy balanced diet. In the weight maintenance phase, a personalised diet was advised based on the estimated energy requirements to maintain body weight.

\section{Physical activity}

The physical activity component was based on guidance of the benefits of being physically active and followed consensus guidelines on physical activity programmes for beginners ${ }^{(31)}$. Participants were supported to gradually increase their participation in physical activity towards aiming to achieve health recommendations on the duration and intensity of physical activity $^{(32,33)}$. This was achieved by setting physical activity goals based on the participants' current level of physical activity, abilities and their preferred form of physical activity. Physical activity goals were individualised for each participant and focused on three types of physical activity:

(1) Lifestyle physical activity: physical activity that could be performed in the home environment such as housework, walking up stairs and following the interactive you can do it DVD.

(2) Walking: based on baseline average steps per day, individuals were encouraged to set targets to progressively increase walking behaviour and used pedometers to monitor step counts.

(3) Sport and exercise: information was given to each participant on local leisure facilities and clubs with accessible sports and exercise groups/classes ${ }^{(16)}$.

\section{Behaviour change techniques}

The most powerful behaviour change techniques shown to support changes in body weight (goal setting, self-monitoring, review of goals and feedback) were used in every session ${ }^{(2,3,34)}$. Specific, Measurable, Relevant, and Time-specific (SMART) ${ }^{(35)}$ goals were set relevant to individual dietary habits and physical activity levels. Participants were encouraged to monitor their food intake (to the specified number of portions of the EDD) and physical activity, in diaries with support from carers. Diaries were reviewed and used to monitor progress, identify potential barriers to change and discuss progress to achieve goals.

\section{Weight maintenance}

To maintain body weight loss participants were encouraged with support from carers, where appropriate, to maintain the healthy lifestyle habits from phase one. Dietary intake followed the same dietary principles used to support weight loss, without an energy deficit of $2510 \mathrm{~kJ} / \mathrm{d}(600 \mathrm{kcal} / \mathrm{d})$. Instead, dietary plans aimed to ensure a euenergetic dietary prescription and intake. Individuals were encouraged to build on the levels of physical activity they achieved in the first 6 months and continue to aim to meet clinical recommendations. Behaviour change techniques used in the weight loss phase were continued to be used flexibly. Specific approaches, in particular, relapse prevention/coping planning and barrier identification/ problem solving were used to prevent large fluctuations in body weight. Weekly self-monitoring of body weight was encouraged as this has been shown to be influential in maintaining body weight. ${ }^{(36)}$ Regular self-monitoring also helped to implement this behaviour as part of their routine in order to facilitate weight maintenance after the programme had finished.

\section{Waist Winners Too components}

WWToo was developed from the original mainstream Waist Winners weight management programme by a partnership 
group of health professionals, NHS Dietitians, Learning Disabilities Nursing, Health Improvement and Glasgow Life. For the purpose of this study, the format was adapted from the original community group programme with eight weekly sessions to an individualised programme, delivered on a one-to-one basis. Participants in this comparator programme received the same number of sessions as participants in the TAKE 5.

\section{Diet}

The dietary component of the programme focused on a health education approach. This was based on the principles of a healthy balanced diet. Non-quantitative dietary advice was provided based on the EatWell Plate ${ }^{(30)}$. Food and drink was categorised as 'healthy' such as fruit and vegetables, 'unhealthy' such as food high in fat and sugar, and other 'healthy' foods such as carbohydrates and dairy products which were advised to be consumed in portion-controlled amounts.

\section{Physical activity}

Physical activity was discussed based on current public health recommendations on increasing activity and reducing sedentary behaviour $^{(3)}$. At each session, participants reviewed their current participation in physical activity and set new goals to increase physical activity levels.

\section{Behaviour change techniques}

The focus of the programme session was to provide educational information on healthy lifestyle behaviours which was achieved by the inclusion of behaviour change techniques. The primary techniques included in each session were goal setting (diet and physical activity goals), self-monitoring (of weight, diet and physical activity) and feedback on performance.

\section{Weight maintenance}

To retain participants to the study for the same duration period as those allocated to TAKE 5, a weight maintenance phase for WWToo was developed. Each session focused on the retention of knowledge delivered in the first phase of the programme. Support was also provided for continued monitoring of diet, physical activity and body weight and an opportunity for questions related to maintaining body weight addressed.

\section{Outcome measures}

A researcher (L. H.) who was blind to study group allocation was responsible for collecting all outcome measures, completed at baseline, at 6 and 12 months. Level of intellectual disabilities, demographic and health questionnaires were completed at the baseline visit. Level of intellectual disabilities was assessed based on questions on participants' ability and development in five key areas of functioning: eating and drinking, intimate care, personal safety, communication and decision making. Based on their score participants were categorised as having mild, moderate, severe or profound level of intellectual disabilities. Total scores assessed by the ability and development questionnaire have shown to be highly associated with the
Vineland's Adaptive Behaviour Scale a validated assessment of functioning and ability level ${ }^{(37)}$ and the questionnaire has been previously used as a measure of level of intellectual disabilities ${ }^{(8,38)}$. Information on the participant's health conditions were self-reported as was information on medication use. Medication was classified as obesogenic based on the association with weight gain $^{(39)}$.

\section{Primary outcome}

The primary outcome measure was the mean difference in body weight $(\mathrm{kg})$ at 12 months from baseline between the two treatment groups.

\section{Secondary outcomes}

Secondary anthropometric outcomes included weight loss of $5 \%$ or more of initial body weight, change in BMI, waist circumference and percentage body fat. A clinically important weight loss was defined as a weight loss of $\geq 5 \%$ of initial body weight based on clinical recommendations ${ }^{(2,3)}$. A weight loss of $<3 \%$ was considered a small weight fluctuation following the recommendation by Stevens et al. ${ }^{(40)}$ Successful weight maintenance was achieved if participants maintained $\pm 2.99 \%$ of their weight loss attained at the end of the weight loss phase. Physical activity outcomes included mean percentage time per day spent engaged in moderate-vigorous intensity physical activity, light intensity physical activity, engagement in sedentary behaviour and total metabolic equivalent-min/week. Nutritional outcomes were not assessed primarily due to the difficulties in accurately and reliably assessing dietary intake in adults with intellectual disabilities ${ }^{(41)}$.

\section{Anthropometric outcomes}

Measurements were made with the participant wearing light clothes without shoes. All measurements were made in duplicate and the final value calculated as the mean of the two measurements. Weight in $\mathrm{kg}$ was measured to the nearest $100 \mathrm{~g}$, using SECA877 scales (SE approval class III; SECA). Height in metres (m) was measured to the nearest $1 \mathrm{~mm}$ using the SECA Leicester stadiometer (SECA). The height $(\mathrm{m})$ and weight $(\mathrm{kg})$ were used to calculate BMI using the formula BMI $=$ weight $/$ height $\left(\mathrm{kg} / \mathrm{m}^{2}\right)$. Waist circumference was measured to the nearest $0.5 \mathrm{~cm}$ at the midpoint between the iliac crest and the lowest rib, in full expiration whilst the participant was standing ${ }^{(42)}$. Percentage body fat was calculated using the triceps skinfold thickness ( $\mathrm{mm}$ ) measured to the nearest $1 \mathrm{~mm}$, waist circumference $(\mathrm{cm})$ and age (years) of the participant. Separate regression equations for male and female participants, developed by Lean et $a l .{ }^{(43)}$ were used to predict body density and percentage body fat.

\section{Physical activity outcomes}

Actigraph GT3X+ accelerometers (Manufacturing Technology Inc.) were worn at the hip, attached to a belt worn round the waist for $7 \mathrm{~d}$. Instructions were given to wear the actigraph during all waking hours, except when showering, bathing or swimming. Validity of accelerometer data, the minimum data requirement, was set at $6 \mathrm{~h}$ of data, on at least $3 \mathrm{~d}$ from $7^{(44)}$. 
The accelerometers were set to record activity over $15 \mathrm{~s}$ intervals (epochs), with activity counts of four consecutive epochs summed to give activity counts per min. This was then categorised as four levels of activity intensity (sedentary, light, moderate and vigorous) based on cut points from previous studies $^{(45)}$. In addition, physical activity levels were also measured subjectively by administering the International Physical Activity Questionnaire-Short (IPAQ-S) ${ }^{(46)}$. In circumstances where participants with intellectual disabilities were regarded by the researcher as unable to answer questions regarding the frequency and time of events, commonly reported as a difficulty for adults with intellectual disabilities ${ }^{(47)}$, carers were invited to assist the participant. To provide an overall measure of physical activity the IPAQ-S variables were combined to calculate total metabolic equivalent-min/ week $^{(48)}$.

\section{Health-related quality of life}

The European Quality of Life-5 dimensions (EQ-5D) youth version was used to measure changes in quality of life $(\mathrm{QOL})^{(49)}$. Completion of generic measures, such as the EQ-5D, can be difficult for adults with intellectual disabilities because of the level of communication and abstraction required. Therefore, for participants with more severe and profound intellectual disabilities, proxy response of health-related QOL was completed from carers.

\section{Serious adverse events}

Serious adverse events (SAE) were defined as an adverse event (an injury or newly diagnosed health condition) that induced hospitalisation or prolonged hospitalisation, resulted in persistent/ significant disability or incapacity, or is life threatening or fatal.

\section{Statistical analysis}

The primary efficacy analysis was conducted according to the intention to treat (ITT) principle. Mixed linear models were used for continuous outcomes to account for clustering and were adjusted for randomised group, baseline variable, and variables used in the minimisation algorithm (level of intellectual disabilities, number of participants in cluster, and presence of Down's syndrome). Data are presented as adjusted mean differences and 95\% CI, corresponding $P$ values and ICC. A logistic regression model was fitted for the categorical outcomes (e.g. weight loss of $5 \%$ or more of initial body weight) taking account of clustering and baseline adjustments listed above. Exploratory, per-protocol analysis was also conducted to examine the validity of the ITT results and examine the efficacy of the programmes under ideal conditions (defined as attendance at $\geq 75 \%$ of sessions). All statistical data were analysed using SPSS 21 IBM statistical package (SPSS IBM).

\section{Results}

Participants were recruited between February and October 2014 (Fig. 1). Recruitment rate was on average six participants per month. The recruitment period could have been extended but due to the limited demand capacity of the dietitian and health professional in terms of scheduling participant appointments, enrolment of participants was spaced out over the recruitment period. Further time restrictions to the recruitment period were introduced as this study was undertaken as part of a $\mathrm{PhD}$ research project and thus completion of this study was necessary within a set timeframe.

\section{Baseline characteristics}

A total of fifty participants were randomised. Participants had mild $(28.0 \%)$, moderate $(42 \cdot 0 \%)$, severe $(22 \cdot 0 \%)$ and profound (8.0\%) level of intellectual disabilities. Eight participants had Down's syndrome as their diagnosis of intellectual disabilities and two participants were diagnosed with Fragile X Syndrome. Four participants were diagnosed with type 2 diabetes, this was, in general, not treated with medication, except for one participant. Participant characteristics and demographics were comparable between study arms (Table 1). All participants were obese and engaged in low levels of physical activity and a large proportion of time spent in sedentary behaviour (Table 2).

\section{Primary outcome}

Based on the ITT analysis there was no significant between group effect on change in body weight post programme at 12 months from baseline (adjusted mean difference $-1.90 \mathrm{~kg}$; $95 \% \mathrm{CI}-4.80,1.01 ; P=0.195$; Table 3 ). There was no evidence of clustering for the primary outcome $(\mathrm{ICC}=0 \cdot 000)$. The within group change in body weight at 12 months revealed a significant programme effect for TAKE $5(-3.55 \mathrm{~kg}$; $95 \% \mathrm{CI}-5.59$, 1.52; $P=0.001)$, however, this was not evident in the WWToo programme $(-1.66 \mathrm{~kg} ; 95 \% \mathrm{CI}-3.69,0.38 \mathrm{~kg} ; P=0.108)$.

\section{Secondary outcomes}

Clinically important changes in body weight. Table 4 illustrates the number of participants achieving a clinically significant weight loss at 6 and 12 months, between the weight management groups. At 12 months, there was a trend for more participants in the TAKE 5 programme $(50.0 \%)$ to achieve a clinically important weight loss than the comparator programme (20.8\%) (OR 3.76; $95 \%$ CI 0.92, 15.30; 0.064). During the weight maintenance phase, the majority of participants in both programmes maintained their weight $(58.3 \%, 68.2 \%$, TAKE 5 and WWToo, respectively) within $\pm 2.99 \%$ of initial body weight. Seven (29.2\%) participants in the TAKE 5 programme and four participants in the WWToo programme (18.2\%) continued to lose weight and three participants in both programme groups gained weight. There was no significant difference in percentage weight change in the weight maintenance phase between programme groups. Descriptive results on percentage weight change categorised as $\geq 10 \%$ is presented in the online Supplementary Table S1.

\section{Anthropometric outcomes}

There was no between group changes in anthropometric outcomes. However, TAKE 5 was associated with significant changes in anthropometric outcomes (weight, BMI, waist circumference 


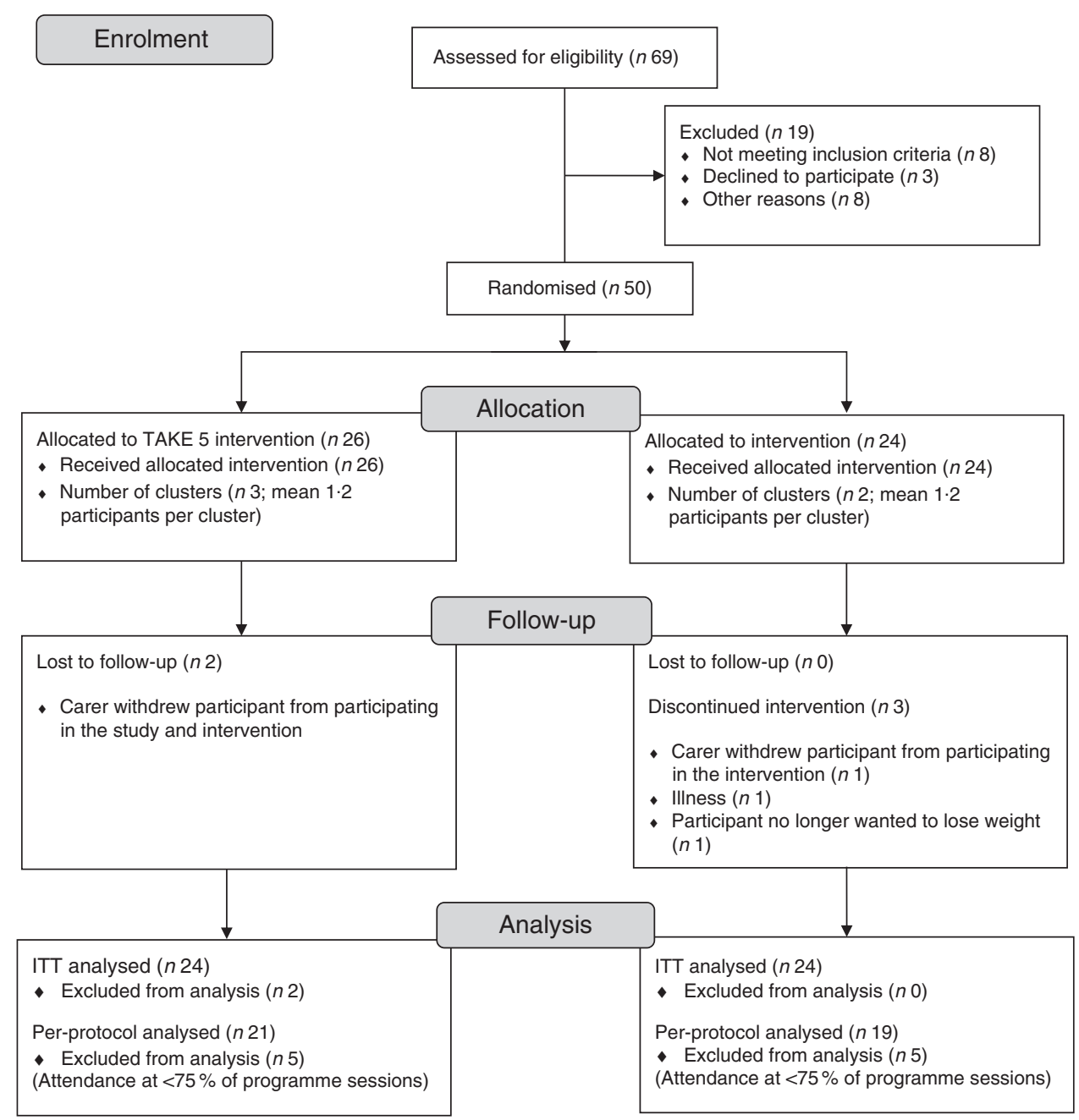

Fig. 1. CONSORT diagram. Flow diagram for the study: outlining the enrolment, screening, allocation and follow-up of participants. Adapted from Schulz et al. ${ }^{(50)}$.

and percentage body fat) at both 6 and 12 months (Table 3). No significant changes were found in the WWToo programme.

\section{Physical activity}

Valid objective accelerometer data were collected for $94 \%$ of participants at baseline. This decreased to 76 and $63 \%$ at 6 and 12 months, respectively. There was no significant between group effect for any of the secondary physical activity outcomes. Results are illustrated in Table 3. Results of the subjective assessment of total physical (IPAQ-S) are not presented due to reliability issues of this measurement.

\section{Health-related quality of life}

A high proportion of participants reported to be in a good health state (EQ-5D index for all participants 0.7 (SD 0.3)). There was no change in health-related QOL at 6 or 12 months from baseline in either the TAKE 5 or the WWToo programme (Table 3).

\section{Per-protocol analysis}

Exploratory analysis on anthropometric outcomes for individuals defined as completing the programme (per-protocol population), revealed similar effect sizes for within group and between group analysis as the ITT analysis (online Supplementary Table S2).

\section{Serious adverse events}

There were no adverse events associated with the trial.

\section{Discussion}

This study contributed to the limited evidence of randomised controlled trials of multi-component weight management programme in adults with intellectual disabilities which adhere to clinical guidelines on the treatment of obesity. This study has demonstrated the successful recruitment and retention of adults with intellectual disabilities to a randomised controlled trial and provided evidence on the feasibility of TAKE 5. Key facilitators and barriers to implementing the programme, and process evaluation including the recruitment, reach and fidelity of the programmes ${ }^{(51)}$ will be published separately.

This study overcame the barriers of recruiting adults with intellectual disabilities reported by previous research ${ }^{(52,53)}$, 
Table 1. Baseline characteristics of participants by randomised group and overall

(Mean values and standard deviations; numbers and percentages)

\begin{tabular}{|c|c|c|c|c|}
\hline \multirow[b]{2}{*}{ Characteristics } & \multicolumn{2}{|c|}{ TAKE 5 (26) } & \multicolumn{2}{|c|}{ WWToo (24) } \\
\hline & $n$ & $\%$ & $n$ & $\%$ \\
\hline \multicolumn{5}{|l|}{ Age (years) } \\
\hline Mean & \multirow{2}{*}{\multicolumn{2}{|c|}{$\begin{array}{l}40 \cdot 6 \\
15 \cdot 0\end{array}$}} & \multicolumn{2}{|c|}{$43 \cdot 6$} \\
\hline SD & & & & \\
\hline \multicolumn{5}{|l|}{ Sex } \\
\hline Males & 8 & $30 \cdot 8$ & 10 & 41.7 \\
\hline Females & 18 & $69 \cdot 2$ & 14 & $58 \cdot 3$ \\
\hline \multicolumn{5}{|l|}{ Ethnicity } \\
\hline Caucasian & 26 & $100 \cdot 0$ & 22 & $91 \cdot 7$ \\
\hline Other Asian background & 0 & 0.0 & 2 & $8 \cdot 3$ \\
\hline \multicolumn{5}{|l|}{ Marital status } \\
\hline Married/live with a partner & 1 & 3.8 & 0 & 0.0 \\
\hline Separated/divorced & 1 & 3.8 & 0 & 0.0 \\
\hline Single & 24 & $92 \cdot 3$ & 24 & $100 \cdot 0$ \\
\hline \multicolumn{5}{|l|}{ SIMD (\% living in quintiles) } \\
\hline 1 (most deprived) & 12 & $46 \cdot 2$ & 9 & 37.5 \\
\hline 2 & 6 & $23 \cdot 1$ & 5 & $20 \cdot 8$ \\
\hline 3 & 2 & $7 \cdot 7$ & 4 & $16 \cdot 7$ \\
\hline 4 & 5 & $19 \cdot 2$ & 5 & $20 \cdot 8$ \\
\hline 5 (least deprived) & 1 & $3 \cdot 8$ & 1 & 4.2 \\
\hline \multicolumn{5}{|l|}{ Social support } \\
\hline Lives independently & 10 & $28 \cdot 4$ & 8 & $33 \cdot 3$ \\
\hline Family carer & 8 & $30 \cdot 8$ & 8 & $33 \cdot 3$ \\
\hline Paid carer & 8 & $30 \cdot 8$ & 8 & $33 \cdot 3$ \\
\hline \multicolumn{5}{|l|}{ Intellectual disabilities } \\
\hline Mild & 8 & $30 \cdot 8$ & 6 & $25 \cdot 0$ \\
\hline Moderate & 11 & $42 \cdot 3$ & 10 & $41 \cdot 7$ \\
\hline Severe & 4 & $15 \cdot 4$ & 7 & $29 \cdot 2$ \\
\hline Profound & 3 & 11.5 & 1 & 4.2 \\
\hline Down's syndrome & 4 & $15 \cdot 4$ & 4 & $16 \cdot 7$ \\
\hline \multicolumn{5}{|l|}{ Health } \\
\hline Epilepsy, seizures or fits & 6 & $23 \cdot 1$ & 5 & $20 \cdot 8$ \\
\hline Vision impairment & 16 & 61.5 & 9 & 37.5 \\
\hline Hearing impairment & 6 & $23 \cdot 1$ & 3 & $12 \cdot 5$ \\
\hline Mental health problems & 6 & $23 \cdot 1$ & 3 & $12 \cdot 5$ \\
\hline Problem behaviour & 10 & 38.5 & 9 & 37.5 \\
\hline High blood pressure & 12 & $46 \cdot 2$ & 11 & $45 \cdot 8$ \\
\hline Obesogenic medication & 7 & $26 \cdot 9$ & 8 & $33 \cdot 3$ \\
\hline Type 2 diabetes & 1 & 3.8 & 3 & 12.5 \\
\hline
\end{tabular}

WWToo, Waist Winners Too; SIMD, Scottish Index of Multiple Deprivation.

Table 2. Baseline primary and secondary outcomes by randomised group (Mean values and standard deviations)

\begin{tabular}{|c|c|c|c|c|c|c|}
\hline \multirow[b]{2}{*}{ Outcomes } & \multicolumn{3}{|c|}{ TAKE 5} & \multicolumn{3}{|c|}{ WWToo } \\
\hline & $n$ & Mean & SD & $n$ & Mean & SD \\
\hline \multicolumn{7}{|l|}{ Primary outcome } \\
\hline Weight $(\mathrm{kg})$ & 26 & $102 \cdot 3$ & $25 \cdot 4$ & 24 & 104.1 & 28.9 \\
\hline \multicolumn{7}{|l|}{ Secondary outcomes } \\
\hline $\mathrm{BMI}\left(\mathrm{kg} / \mathrm{m}^{2}\right)$ & 26 & $40 \cdot 2$ & $6 \cdot 8$ & 24 & 41.2 & $8 \cdot 1$ \\
\hline Waist circumference $(\mathrm{cm})$ & 24 & 121.9 & 14.0 & 23 & $122 \cdot 2$ & $16 \cdot 1$ \\
\hline Percentage body fat $(\%)$ & 24 & 49.3 & $9 \cdot 1$ & 19 & $51 \cdot 7$ & 8.5 \\
\hline Light PA (\% time spent/d) & 25 & $21 \cdot 8$ & $6 \cdot 2$ & 22 & $22 \cdot 3$ & 8.0 \\
\hline MVPA (\% time spent/d) & 25 & 4.5 & $2 \cdot 7$ & 22 & 4.7 & 3.8 \\
\hline $\begin{array}{l}\text { Sedentary behaviour } \\
(\% \text { time spent } / \mathrm{d})\end{array}$ & 25 & 73.7 & 7.6 & 22 & $73 \cdot 0$ & $9 \cdot 7$ \\
\hline EQ-5D index & 26 & 0.8 & 0.3 & 24 & 0.7 & 0.3 \\
\hline
\end{tabular}

WWToo, Waist Winners Too; PA, physical activity; MVPA, moderate to vigorous physical activity; MET: metabolic equivalent; EQ-5D: European Quality of Life-5 dimensions.

evidenced by the minimal participation of this population group in research ${ }^{(52)}$ and the small sample sizes of previous weight management programmes ${ }^{(11-13)}$. Challenges to recruiting adults with intellectual disabilities have been reported including the inability to have direct contact with participants and procedures of taking informed consent ${ }^{(53,54)}$. Effective recruitment in this study was achieved by the identification of a key worker/carer known to the potential participant, and by providing a personal approach to recruitment, for example meeting participants and carers in person, building rapport and aiming to eliminate potential barriers to participating in a research study ${ }^{(27)}$. Recruitment of participants to the study fell short of the projected final sample size of sixty-six participants ${ }^{(22)}$. However, the decision to stop recruitment was justified based on the following reasons: the limited time capacity of the dietitian and health professional to schedule participant appointments, the requirements of the completion of the trial within the researchers $\mathrm{PhD}$ timeframe, and the study had successfully provided sufficient insight into the feasibility of the recruitment and retention rates which would inform a full-scale trial.

The results of this study extend the findings of the singlestranded studies by Melville and colleagues ${ }^{(16,18)}$ by providing further support for the acceptability and feasibility of a multicomponent weight management programme involving an EDD and tailored to meet the individual needs of adults with intellectual disabilities. The exploratory efficacy analysis in this study revealed that TAKE 5 achieved a slightly lower absolute weight loss and clinically important weight loss of $5-10 \%$ of initial body weight $(-2.93 \mathrm{~kg}, 20.5 \%$ v. $-4.47 \mathrm{~kg}, 36.2 \%$, respectively) in comparison to the single-stranded study ${ }^{(16)}$. However, adhering to clinical recommendations, the efficacy of a weight management programme should be assessed at 12 months or more ${ }^{(2,3)}$. At the end of the weight maintenance phase and end of the programme, participants in TAKE 5 in this study had continued to lose weight (overall weight change from baseline at 12 months: $-3.55 \mathrm{~kg}$ ). This may reflect the design of this study. If participants had not achieved a clinically significant weight loss of $5 \%$ at 6 months they were offered the option to continue to lose weight for a further 3 months, followed by 3 months of weight maintenance. The weight loss in this study compares favourably with the changes in body weight in the single-stranded weight maintenance phase in which participants did not maintain a significant reduction in body weight, $-0.6 \mathrm{~kg}$. Based on the definitions by Stevens et al. ${ }^{(40)}, 58 \%$ of participants maintained their weight loss in this study in comparison to $50 \%$ of participants in the single-stranded study ${ }^{(18)}$. Moreover, in this study fewer participants gained weight (13v . $29 \%$ ) and a higher percentage of participants continued to lose weight (29 v. 21\%). Although, these results should be interpreted cautiously due to the differences in the duration of the weight maintenance periods (12 months ${ }^{(18)}$ in comparison to 6 months weight maintenance phase in this study), overall the results suggest that TAKE 5 can achieve sustainable clinically important changes in body weight.

\section{Clinically important weight loss}

An important finding of this study is that the degree of weight loss achieved in TAKE 5 is comparable to the weight loss achieved in the general population on completion of the GCWMS programme ${ }^{(20)}$. Mean weight change at 12 months for 
Table 3. Change in primary and secondary outcomes at 6 and 12 months from baseline (Mean values and $95 \%$ confidence intervals)

\begin{tabular}{|c|c|c|c|c|c|c|c|c|c|c|c|c|}
\hline \multirow[b]{2}{*}{ Anthropometric outcomes } & \multicolumn{4}{|c|}{ TAKE 5} & \multicolumn{4}{|c|}{ WWToo } & \multicolumn{4}{|c|}{ Difference between groups } \\
\hline & $n$ & Mean* & $95 \% \mathrm{Cl}$ & $P$ & $n$ & Mean* & $95 \% \mathrm{Cl}$ & $P$ & Mean* & $95 \% \mathrm{Cl}$ & $P$ & ICC \\
\hline \multicolumn{13}{|l|}{ Change in weight $(\mathrm{kg})$} \\
\hline 6 months & 24 & -2.93 & $-4 \cdot 42,-1.44$ & $<0.001$ & 22 & $-1 \cdot 26$ & $-2 \cdot 82,0 \cdot 30$ & 0.110 & -1.67 & $-3 \cdot 84,0.50$ & 0.126 & 0.059 \\
\hline 12 months & 24 & -3.55 & $-5.59,-1.52$ & 0.001 & 24 & -1.66 & $-3.69,0.38$ & 0.108 & -1.90 & $-4 \cdot 80,1 \cdot 01$ & 0.195 & 0.000 \\
\hline \multicolumn{13}{|l|}{ Change in weight (\%) } \\
\hline 6 months & 24 & $-3 \cdot 25$ & $-4 \cdot 81,-1 \cdot 69$ & $<0.001$ & 22 & -0.98 & $-2 \cdot 61,0.65$ & 0.231 & $-2 \cdot 27$ & $-4.54,0.00$ & 0.050 & 0.000 \\
\hline 12 months & 24 & $-3 \cdot 80$ & $-5 \cdot 86,-1 \cdot 74$ & 0.001 & 24 & $-1 \cdot 22$ & $-3.28,0.83$ & 0.237 & $-2 \cdot 58$ & $-5 \cdot 52,0.36$ & 0.084 & 0.000 \\
\hline \multicolumn{13}{|l|}{ Change in BMI (kg/m2) } \\
\hline 6 months & 24 & $-1 \cdot 19$ & $-1 \cdot 77,-0.62$ & $<0.001$ & 22 & -0.46 & $-1 \cdot 06,0 \cdot 15$ & 0.133 & -0.74 & $-1 \cdot 58,0 \cdot 11$ & 0.085 & 0.000 \\
\hline 12 months & 24 & -1.48 & $-2 \cdot 29,-0.66$ & 0.001 & 24 & -0.59 & $-1.41,0.23$ & $0 \cdot 154$ & -0.89 & $-2 \cdot 05,0.28$ & $0 \cdot 134$ & 0.000 \\
\hline \multicolumn{13}{|c|}{ Change in waist circumference $(\mathrm{cm})$} \\
\hline 6 months & 22 & $-3 \cdot 15$ & $-4.91,-1.40$ & 0.001 & 20 & -1.45 & $-3 \cdot 29,0.40$ & $0 \cdot 120$ & $-1 \cdot 71$ & $-4 \cdot 28,0.86$ & 0.186 & 0.176 \\
\hline 12 months & 22 & -3.60 & $-5 \cdot 99,-1 \cdot 21$ & 0.004 & 21 & $-1 \cdot 83$ & $-4 \cdot 24,0.58$ & $0 \cdot 132$ & $-1 \cdot 77$ & $-5 \cdot 20,1 \cdot 67$ & 0.304 & 0.267 \\
\hline \multicolumn{13}{|c|}{ Change in percentage body fat (\%) } \\
\hline 6 months & 22 & $-1 \cdot 79$ & $-3.08,-0.50$ & 0.008 & 18 & -1.02 & $-2 \cdot 45,0.41$ & $0 \cdot 155$ & -0.77 & $-2 \cdot 72,1 \cdot 19$ & 0.430 & 0.187 \\
\hline 12 months & 22 & $-2 \cdot 23$ & $-3.95,-0.51$ & 0.013 & 18 & -0.65 & $-2 \cdot 56,1 \cdot 26$ & 0.493 & -1.58 & $-4 \cdot 21,1 \cdot 05$ & 0.231 & 0.000 \\
\hline \multicolumn{13}{|c|}{ Sedentary behaviour (\% time spent/d) } \\
\hline 6 months & 20 & -2.08 & $-0.27,4 \cdot 43$ & 0.080 & 15 & $-2 \cdot 00$ & $-0.67,4.67$ & $0 \cdot 136$ & -0.09 & $-3.50,3 \cdot 67$ & 0.962 & 0.450 \\
\hline 12 months & 16 & -0.91 & $-4 \cdot 05,2 \cdot 24$ & 0.556 & 13 & 1.05 & $-2 \cdot 33,4 \cdot 42$ & 0.526 & -1.95 & $-6 \cdot 61,2 \cdot 70$ & 0.394 & 0.994 \\
\hline \multicolumn{13}{|l|}{ Light PA (\% time spent/d) } \\
\hline 6 months & 20 & -1.79 & $-3.69,0.11$ & 0.064 & 15 & $-1 \cdot 22$ & $-3.40,0.96$ & 0.262 & -0.57 & $-3 \cdot 50,2 \cdot 35$ & 0.692 & 0.164 \\
\hline 12 months & 16 & 0.79 & $-2 \cdot 22,3 \cdot 81$ & 0.591 & 13 & -0.92 & $-4 \cdot 15,2 \cdot 31$ & 0.561 & $1 \cdot 71$ & $-2 \cdot 75,6 \cdot 17$ & 0.434 & 0.994 \\
\hline \multicolumn{13}{|l|}{ MVPA (\% time spent/d) } \\
\hline 6 months & 20 & -0.32 & $-1 \cdot 17,0.54$ & 0.455 & 15 & -0.81 & $-1 \cdot 77,0 \cdot 15$ & 0.093 & 0.50 & $-0 \cdot 79,1 \cdot 78$ & 0.434 & 0.895 \\
\hline 12 months & 16 & 0.10 & $-0.94,1.13$ & 0.849 & 13 & -0.17 & $-1.28,0.95$ & 0.758 & 0.26 & $-1 \cdot 28,1 \cdot 80$ & 0.726 & 0.818 \\
\hline \multicolumn{13}{|l|}{ Total (\% time spent/d) } \\
\hline 6 months & 20 & -2.08 & $-0.27,4.43$ & 0.079 & 15 & $-2 \cdot 00$ & $-0.67,4.67$ & 0.137 & -0.09 & $-3.50,3.67$ & 0.962 & 0.449 \\
\hline 12 months & 16 & 0.91 & $-2.24,4.05$ & 0.556 & 13 & -1.05 & $-4 \cdot 42,2 \cdot 33$ & 0.526 & 1.95 & $-2 \cdot 70,6 \cdot 61$ & 0.394 & 0.994 \\
\hline \multicolumn{13}{|l|}{ EQ-5D index } \\
\hline 6 months & 24 & 0.07 & $-0.03,0.17$ & 0.177 & 22 & 0.04 & $-0.07,0.14$ & 0.500 & 0.03 & $-0.12,0.18$ & 0.652 & 0.118 \\
\hline 12 months & 24 & 0.00 & $-0.14,0.14$ & 0.977 & 24 & -0.04 & $-0 \cdot 18,0 \cdot 10$ & 0.569 & 0.04 & $-0.16,0.24$ & 0.675 & 0.000 \\
\hline
\end{tabular}

WWToo, Waist Winners Too; ICC, interclass correlation coefficient; MVPA, moderate to vigorous physical activity; ED-5D, European Quality of Life-5 dimensions.

* Adjusted for cluster, baseline value and stratification variables (number of participants within a cluster, level of intellectual disability and presence of Down's syndrome).

Table 4. Percentage weight change at 6 and 12 months from baseline (Numbers and percentages; odds ratios and $95 \%$ confidence intervals)

\begin{tabular}{|c|c|c|c|c|c|c|c|c|c|}
\hline \multirow[b]{2}{*}{ Percentage weight change } & \multicolumn{3}{|c|}{ TAKE 5} & \multicolumn{3}{|c|}{ WWToo } & \multirow[b]{2}{*}{ OR } & \multirow[b]{2}{*}{$95 \% \mathrm{Cl}$} & \multirow[b]{2}{*}{$P$} \\
\hline & $n$ & $n$ & $\%$ & $n$ & $n$ & $\%$ & & & \\
\hline Weight loss phase (0-6 months) & 24 & & & 22 & & & & & \\
\hline Weight loss $>5 \%$ & & 5 & $20 \cdot 8$ & & 2 & $9 \cdot 1$ & $2 \cdot 70$ & $0.44,16.59$ & 0.275 \\
\hline Weight loss $<5 \%$ & & 19 & 79.2 & & 20 & $90 \cdot 9$ & & Ref. & \\
\hline Weight maintenance phase (6-12 months) & 24 & & & 22 & & & & & \\
\hline loss $>3 \%$ & & 7 & $29 \cdot 2$ & & 4 & $18 \cdot 2$ & 1.57 & $0 \cdot 18,13 \cdot 90$ & 0.679 \\
\hline Weight maintenance $( \pm 2.99 \%)$ & & 14 & $58 \cdot 3$ & & 15 & $68 \cdot 2$ & 0.92 & $0 \cdot 14,5 \cdot 91$ & 0.924 \\
\hline Weight gain >3\% & & 3 & 12.5 & & 3 & 13.6 & & Ref. & \\
\hline Weight loss + weight maintenance (0-12 months) & 24 & & & 24 & & & & & \\
\hline Weight loss > $\%$ & & 12 & $50 \cdot 0$ & & 5 & $20 \cdot 8$ & 3.76 & $0.92,15 \cdot 30$ & 0.064 \\
\hline Weight loss $<5 \%$ & & 12 & $50 \cdot 0$ & & 19 & $79 \cdot 2$ & & Ref. & \\
\hline
\end{tabular}

WWToo, Waist Winners Too; Ref. referent values.

* Adjusted for cluster, and stratification variables (number of participants within a cluster, level of intellectual disability and presence of Down's syndrome).

the general population was $-3.6 \mathrm{~kg}(95 \% \mathrm{CI}-3.9,-3 \cdot 3)$ in comparison with $-3.6 \mathrm{~kg}(95 \% \mathrm{CI}-5 \cdot 6,-1 \cdot 5)$ of participants in TAKE 5. Furthermore, the proportion of participants achieving a clinically important weigh loss was greater in the TAKE 5 programme (50\%) in comparison to the participants in the GCWMS (24\%). This illustrates that adults with intellectual disabilities, can access evidence-based weight management services which are tailored to their needs and achieve clinically important weight losses, comparable with the general population.

\section{Programme components}

The study design of this trial facilitated the direct comparison of programme components between the two weight management 
programmes. As both programmes were similar except for the differences in the diet component (TAKE 5: quantitative EDD; WWTOO: non-quantitative dietary advice) the potential efficacy of TAKE 5 can be attributed to the EDD. Although, physical activity plays an integral role in weight loss and the maintenance of body weight, the physical activity component in both weight management programmes ${ }^{(2,3)}$ failed to elicit an effect on increasing physical activity or reducing sedentary behaviour. Adults with intellectual disabilities experience significant barriers to engaging in physical activity including; a reduced cognitive ability to understand the benefits of physical activity, a lack of awareness of physical activity options, limited time capacity and support from carers to assist engagement in physical activity and limitations in transportation ${ }^{(55,56)}$. Possible explanations for participants not achieving their physical activity goals were associated with the environment and concerns over safety in performing physical activity outside the home. In all, $52 \%$ of participants lived in the most deprived areas of Glasgow and therefore may have limited opportunities to increase physical activity. This is in agreement with previous research investigating the barriers and facilitators to physical activity $^{(57,58)}$. Melville et al $^{(58)}$ attributed the ineffectiveness of their walking programme in part to the unsupportive environment. Moreover, participants engaged in high levels of sedentary behaviour, and previous research has shown that individuals have low levels of cardiorespiratory fitness ${ }^{(59,60)}$. Therefore, participants may not have been able to tolerate physical activity levels required to improve health ${ }^{(32,33)}$. Another potential explanation which could in part explain the limited changes in physical activity could also be related in the challenges in adapting complex programmes for the needs of this population group ${ }^{(61)}$. Behaviour change techniques implemented to support increased physical activity were feedback, goal setting and self-monitoring. Although shown to be effective in changing dietary habits, these may not have been of significant magnitude to change levels of physical activity. Future implementation of the programme should refine the physical activity component to focus on feasible home-based activities, interrupting sedentary behaviour and engaging carers in the implementation of the programme. The following strategies, shown to be effective in the general population, should also be utilised to support a change in physical activity behaviour; incorporating the practice of physical activity into the sessions, providing prompts cues to stimulate increased physical activity between sessions, and encourage peers, family and friends to engage in this lifestyle behaviour ${ }^{(62,63)}$. Enhancement of these programme components, in particular the support to increase physical activity, in addition to the already positive changes in diet, could provide a larger effect size on weight loss.

\section{Comparison with previous literature}

Comparison of the results of this study with previous weight management programmes based on a standard health education approach ${ }^{(11-13,21)}$ confirms that a health education approach to weight management is not effective. No significant improvements in outcomes were found at 6 and 12 months. Although a proportion of the participants in WWToo achieved a clinically important weight loss of $5 \%$, this may be explained by the increased intensity of the WWToo programme in comparison with previous health education programmes. The number of sessions, duration of the programme and weight maintenance component were added to control for contact time and do not reflect clinical health education approaches. This is consistent with clinical recommendations in the general population $^{(2,3)}$, which do not support generalised health education approaches for the treatment of obesity, as these are not prescriptive in energy intake and therefore more open to interpretation by the individual.

The results of this study are in agreement with the available literature demonstrating that multi-component weight management programmes including an $\mathrm{EDD},{ }^{(14,16,18,64,65)}$ and specifically tailored to the needs of adults with intellectual disabilities are acceptable and support clinically meaningful weight loss. For example, a recent study by Ptomey et al. ${ }^{(65)}$ compared an enhanced stop light diet based on pre-prepared portioncontrolled meals to a conventional EDD (2092-2929 kJ/d deficit $(500-700 \mathrm{kcal} / \mathrm{d}$ deficit)). In all, $51 \%$ of the participants in the conventional EDD group achieved a clinically important weight loss at 18 months, which is similar to the findings of this study ( $50 \%$ of participants in TAKE 5 ).

\section{Strengths and limitations}

This study adds to the limited evidence of weight management in adult with intellectual disabilities by examining the efficacy of the programmes using a randomised controlled trial design. The results of this study provide a more valid and reliable estimate of the effect of the programme, by aiming to reduce methodological errors often associated with observation studies ${ }^{(25)}$ such as imbalances between participant characteristics under investigation, un-measurable confounding factors and reverse causality. Furthermore, this study included all adults with varying severity of intellectual disabilities. This is the first study to provide weight management to adults with all levels of intellectual disabilities (mild to profound). This highlights with support from carers to implement the programme, this population group irrespective is severity of intellectual disabilities can achieve improvements in body weight and composition.

Reliability of self-report outcomes measures was a limitation of this study. For this reason, data on participant's dietary intake was not assessed. Although, food diaries were used as a behaviour change stagey to self-monitor dietary intake, this was not included as an outcome and therefore prevented insight into compliance with the EDD. Self-report measures of physical activity and health-related QOL were included in this study. Self-report measures of physical activity by the IPAQ-S are dependent on memory to recall physical activity levels over a 7-d period and thought to be close to the limits of the cognitive abilities of many adults with intellectual disabilities ${ }^{(66)}$. Furthermore, comprehension of information on the intensity of physical activities and quantifying amounts of time spent in physical activity was shown to be challenging for some adults with intellectual disabilities. For some participants, support from carers was encouraged to assist in completion of the IPAQ-S. However, issues identified in administering the IPAQ-S were 
the high turnover of carers which meant that some carers were not aware of activities performed over the $7 \mathrm{~d}$. Moreover, participants often attended day centres or clubs and therefore engagement in physical activity out with the home environment was unknown. Participants also found completion of the EQ-5D visual analogue scale, a measure of health-related QOL, challenging and therefore, results were presented solely for the EQ-5D index. The reliability of the proxy response of EQ-5D of carers is also unknown as research on the use of this questionnaire is limited ${ }^{(67)}$ and similarly, a lack of consistency in carers at outcome measure appointments meant that measures were not always completed by the same carer. As proxy response is an integral component to conducting research, and in clinical practice involving adults with intellectual disabilities $^{(66)}$, it is important that future research investigates valid methodologies to accurately and reliably measure self-report physical activity levels and health-related QOL of adults with intellectual disabilities.

\section{Research and clinical recommendations}

This study contributes to the evidence by closing the gap between research and providing accessible weight management services for this population group. This study provides evidence that an EDD is feasible and an acceptable approach to the treatment of obesity. Due to the negative health risks associated with obesity, clinicians and health professionals need to recognise that in order to achieve weight loss with clinically important benefits, current programmes based on a health education approach are ineffective. A full-scale trial is warranted to establish the effectiveness of TAKE 5 modified on the results of this study.

\section{Conclusion}

This study provides evidence on the feasibility of conducting a full-scale trial of a multi-component weight management programme in adults with intellectual disabilities. The multi-point recruitment strategy was shown to be successful in overcoming barriers in conducting research in adults with intellectual disabilities and was able to recruit adults with all levels of intellectual disabilities. This study illustrates the acceptability and feasibility of an EDD approach to weight management in adults with intellectual disabilities and illustrates that current service provision based on health education is not effective in the treatment of obesity. A future full-scale randomised controlled trial is warranted to confirm these findings and provide evidence on the optimum approach to weight management in this population group.

\section{Acknowledgements}

The authors would like to thank the participants and family and paid carers.

This work was supported by the Scottish Government Equally Well Fund.

L. H., C. M. and C. H. led the drafting and editing of the manuscript. L. H. acquired and analysed the data. N. J. was the dietitian on the study. C. P., S. B., H. M. and J. T. were all involved in the original application and the design in the study. All authors read and approved the final manuscript.

None of the authors has any conflicts of interest to declare.

\section{Supplementary material}

For supplementary material/s referred to in this article, please visit https://doi.org/10.1017/S0007114517001933

\section{References}

1. National Institutes of Health, National Heart, Lung, and Blood Institute \& North American Association for the Study of Obesity (2000) The Practical Guide Identification, Evaluation, and Treatment of Overweight and Obesity in Adults. Bethesda, MD: NIH.

2. Scottish Intercollegiate Guideline Network (2010) Management of Obesity: A National Clinical Guideline. Edinburgh: SIGN.

3. National Institute for Health and Clinical Excellence (2014) Obesity: Identification, Assessment and Management of Overweight and Obesity in Children, Young People and Adults, CG189. London: NICE.

4. Yumuk V, Tsigos C, Fried M, et al. (2015) European guidelines for obesity management in adults. Obes Facts 8, 402-424.

5. Loveman E, Frampton GK, Shepherd J, et al. (2011) The clinical effectiveness and cost-effectiveness of long-term weight management schemes for adults: a systematic review. Health Technol Assess 15, 1-182.

6. Schalock RL, Borthwick-Duffy SA, Bradley VJ, et al. (2010) Intellectual Disability: Definition, Classification, and Systems of Supports. Washington, DC: American Association on Intellectual and Developmental Disabilities.

7. Melville CA, Hamilton S, Hankey CR, et al. (2007) The prevalence and determinants of obesity in adults with intellectual disabilities. Obes Rev 8, 223-230.

8. Melville CA, Cooper SA, Morrison J, et al. (2008) The prevalence and determinants of obesity in adults with intellectual disabilities. J Appl Res Intellect Disabil 21, 425-437.

9. Bhaumik S, Watson JM, Thorp CF, et al. (2008) Body mass index in adults with intellectual disability: distribution, associations and service implications: a population-based prevalence study. J Intellect Disabil Res 52, 287-298.

10. Hsieh K, Rimmer JH \& Heller T (2014) Obesity and associated factors in adults with intellectual disability. J Intellect Disabil Res 58, 851-863.

11. Hamilton S, Hankey CR, Miller S, et al. (2007) A review of weight loss interventions for adults with intellectual disabilities. Obes Rev 8, 339-345.

12. Jinks A, Cotton A \& Rylance R (2011) Obesity interventions for people with a learning disability: an integrative literature review. J Adv Nurs 67, 460-471.

13. Spanos D, Melville CA \& Hankey CR (2013) Weight management interventions in adults with intellectual disabilities and obesity: a systematic review of the evidence. Nutr $J$ 12, 1-16.

14. Saunders RR, Saunders MD, Donnelly JE, et al. (2011) Evaluation of an approach to weight loss in adults with intellectual or developmental disabilities. Intellect Dev Disabil 49, 103-112.

15. Jackson HJ \& Thorbecke PJ (1982) Treating obesity of mentally retarded adolescents and adults: an exploratory program. Am J Ment Defic 87, 302-308. 
16. Melville CA, Boyle S, Miller S, et al. (2011) An open study of the effectiveness of a multi-component weight-loss intervention for adults with intellectual disabilities and obesity. $\mathrm{Br} \mathrm{J}$ Nutr 105, 1553-1562.

17. Spanos D, Hankey CR, Boyle S, et al. (2013) Carers' perspectives of a weight loss intervention for adults with intellectual disabilities and obesity: a qualitative study. $J$ Intellect Disabil Res 57, 90-102.

18. Spanos D, Hankey CR \& Melville CA (2015) The effectiveness of a weight Maintenance intervention for adults with intellectual disabilities and obesity: a single stranded study. J Appl Res Intellect Disabil 29, 317-329.

19. Morrison DS, Boyle S, Morrison C, et al. (2012) Evaluation of the first phase of a specialist weight management programme in the UK National Health Service: prospective cohort study. Public Health Nutr 15, 28-38.

20. Logue J, Allardice G, Gillies M, et al. (2014) Outcomes of a specialist weight management programme in the UK National Health Service: prospective study of 1838 patients. BMJ Open 4, e003747.

21. Jones N, Melville CA, Tobin J, et al. (2015) A retrospective evaluation of an adapted group weight management intervention for adults with intellectual disabilities: Waist Winners Too. Br J Obes 1, 132-140.

22. Harris L, Melville C, Jones N, et al. (2015) A single-blind, pilot randomised trial of a weight management intervention for adults with intellectual disabilities and obesity: study protocol. Pilot Feasibility Stud 1, 1-12.

23. Campbell MK, Piaggio G, Elbourne DR, et al. (2012) Consort 2010 statement: extension to cluster randomised trials. BMJ 345, e5661.

24. Medical Research Council (2000) A Framework for the Development and Evaluation of RCTs for Complex Interventions to Improve Health. London: MRC.

25. Medical Research Council (2008) Developing and Evaluating Complex Interventions: New Guidance. London: MRC. http:// www.mrc.ac.uk/documents/pdf/complex-interventions-guidance/ (accessed September 2016).

26. Executive S (2000) Adults with Incapacity (Scotland) Act 2000. Edinburgh: The Stationery Office.

27. Foster CE, Brennan G, Matthews A, et al. (2011) Recruiting participants to walking intervention studies: a systematic review. Int J Behav Nutr Phys Act 8, 1.

28. Mifflin MD, St Jeor ST, Hill LA, et al. (1990) A new predictive equation for resting energy expenditure in healthy individuals. Am J Clin Nutr 51, 241-247.

29. Leslie WS, Lean MEJ, Baillie HM, at al. (2002) Weight management: a comparison of existing dietary approaches in a work-site setting. Int J Obes 26, 1469-1475.

30. Food Standards Agency (2009) The Eatwell Plate. London: Food Standards Agency. www.food.gov.uk/ (accessed September 2016)

31. O'Donovan G, Blazevich AJ, Boreham C, et al. (2010) The $\mathrm{ABC}$ of Physical Activity for Health: a consensus statement from the British Association of Sport and Exercise Sciences. J Sports Sci 28, 573-591.

32. Department of Health (2004) At Least Five a Week. Evidence for the Impact of Physical Activity and its Relationship to Health. London: Department of Health.

33. National Health Service (NHS) Health Scotland (2009) Five Year Review of "Let's Make Scotland More Active" - A Strategy for Physical Activity. Edinburgh: NHS.

34. Michie S, Abraham C, Whittington C, et al. (2009) Effective techniques in healthy eating and physical activity interventions: a meta-regression. Health Psychol 28, 690.

35. Doran GT (1981) There's a S.M.A.R.T. way to write management's goals and objectives. Manage Rev 70, 35-36.
36. Wing RR \& Phelan S (2005) Long-term weight loss maintenance. Am J Clin Nutr 82, 222S-225S

37. Sparrow SS, Balla DA \& Cicchetti DV (1984) Vineland Adaptive Behavior Scales. Circle Pines, MN: American Guidance Service.

38. Cooper SA (1997) Epidemiology of psychiatric disorders in elderly compared with younger adults with learning disabilities. Br J Psychiatry 170, 375-380.

39. Domecq JP, Prutsky G, Leppin A, et al. (2015) Drugs commonly associated with weight change: a systematic review and meta-analysis. J Clin Endocrinol Metab 100, 363-370.

40. Stevens J, Truesdale KP, McClain JE, et al. (2006) The definition of weight maintenance. Int J Obes 30, 391-399.

41. Humphries K, Traci MA \& Seekins T (2009) Nutrition and adults with intellectual or developmental disabilities: systematic literature review results. Intellect Dev Disabil 47, 163-185.

42. World Health Organization (2008) WHO STEPwise Approach to Surveillance (STEPS). Geneva: WHO

43. Lean ME, Han TS \& Deurenberg P (1996) Predicting body composition by densitometry from simple anthropometric measurements. Am J Clin Nutr 63, 4-14.

44. Penpraze V, Reilly JJ, MacLean C, et al. (2006) Monitoring of physical activity in young children: how much is enough? Pediatr Exer Sci 18, 483-491.

45. Atkin AJ, Gorely T, Clemes SA, et al. (2012) Methods of measurement in epidemiology: sedentary behaviour. Int $\mathrm{J}$ Epidemiol 41, 1460-1471.

46. Craig CL, Marshall AL, Sjostrom M, et al. (2003) International physical activity questionnaire: 12-country reliability and validity. Med Sci Sports Exerc 35, 1381-1395.

47. Finlay WM \& Lyons E (2001) Methodological issues in interviewing and using self-report questionnaires with people with mental retardation. Psychol Assess 13, 319.

48. Jette M, Sidney K \& Blümchen G (1990) Metabolic equivalents (METS) in exercise testing, exercise prescription, and evaluation of functional capacity. Clin Cardiol 13, 555-565.

49. Brooks R \& Group E (1996) EuroQol: the current state of play. Health Policy 37, 53-72.

50. Schulz KF, Altman DG \& Moher D (2010) CONSORT 2010 statement: updated guidelines for reporting parallel group randomized trials. Ann Intern Med 152, 726-732.

51. Linnan L \& Steckler A (2002) Process Evaluation for Public Health Interventions and Research. San Francisco, CA: Jossey-Bass.

52. Cleaver S, Ouellette-Kuntz H \& Sakar A (2010) Participation in intellectual disability research: a review of 20 years of studies. J Intellect Disabil Res 54, 187-193.

53. Lennox N, Taylor M, Rey-Conde T, et al. (2005) Beating the barriers: recruitment of people with intellectual disability to participate in research. J Intellect Disabil Res 49, 296-305.

54. Iacono $\mathrm{T}$ (2006) Ethical challenges and complexities of including people with intellectual disability as participants in research. J Intellect Dev Disabil 31, 173-179.

55. Hawkins A \& Look R (2006) Levels of engagement and barriers to physical activity in a population of adults with learning disabilities. Br J Learn Disabil 34, 220-226.

56. Bodde AE \& Seo DC (2009) A review of social and environmental barriers to physical activity for adults with intellectual disabilities. Disabil Health J 2, 57-66.

57. Mitchell F, Melville C, Stalker K, et al. (2013) Walk Well: a randomised controlled trial of a walking intervention for adults with intellectual disabilities: study protocol. BMC Public Health 13, 1-13.

58. Melville CA, Mitchell F, Stalker K, et al. (2015) Effectiveness of a walking programme to support adults with intellectual disabilities to increase physical activity: walk well cluster-randomised controlled trial. Int J Behav Nutr Phys Act 12, 1-11. 
59. Rimmer JH, Heller T, Wang E, et al. (2004) Improvements in physical fitness in adults with Down syndrome. Am J Ment Retard 109, 165-174.

60. Oppewal A, Hilgenkamp TI, van Wijck R, et al. (2013) Cardiorespiratory fitness in individuals with intellectual disabilities - a review. Res Dev Disabil 34, 3301-3316.

61. National Institute for Health and Clinical Excellence (2007) Behaviour Change at Population, Community and Individual Levels. London: NICE.

62. Dombrowski SU, Sniehotta FF, Avenell A, et al. (2012) Identifying active ingredients in complex behavioural interventions for obese adults with obesity-related co-morbidities or additional risk factors for co-morbidities: a systematic review. Health Psychol Rev 6, 7-32.

63. Olander EK, Fletcher H, Williams S, et al. (2013) What are the most effective techniques in changing obese individuals' physical activity self-efficacy and behaviour: a systematic review and meta-analysis. Int J Behav Nutr Phys Act 10, 1-15.
64. Martinez-Zaragoza F, Campillo-Martinez JM \& Ato-Garcia M (2015) Effects on physical health of a multicomponent programme for overweight and obesity for adults with intellectual disabilities. I Appl Res Intellect Disabil 29, 250-265.

65. Ptomey LT, Saunders RR, Saunders M, et al. (2017) Weight management in adults with intellectual and developmental disabilities: a randomized controlled trial of two dietary approaches. J Appl Res Intellect Disabil (epublication ahead of print version 23 March 2017).

66. Finlay WM \& Lyons E (2001) Methodological issues in interviewing and using self-report questionnaires with people with mental retardation. Psychol Assess 13, 319-335.

67. Cooper SA, Morrison J, Allan LM, et al. (2014) Practice nurse health checks for adults with intellectual disabilities: a cluster-design, randomised controlled trial. Lancet Psychiatry 1, 511-521. 